\title{
Perceptual processing links autism and synesthesia: a twin study
}

\author{
Tessa M van Leeuwen ${ }^{a}$, Lowe Wilsson ${ }^{b}$, Hjalmar Nobel Norrman ${ }^{b}$, Mark Dingemanse $^{a, c}$, \\ Sven Bölte $e^{b, d, e} \&$ Janina Neufeld ${ }^{b, *}$
}

a) Donders Institute for Brain, Cognition and Behaviour, Radboud University, Nijmegen, The Netherlands

b) Center of Neurodevelopmental Disorders (KIND), Centre for Psychiatry Research, Department of Women's and Children's Health, Karolinska Institutet \& Stockholm Health Care Services, Region Stockholm, Gävlegatan 22, 11330 Stockholm, Sweden

c) Centre for Language Studies, Radboud University, Erasmusplein 1, 6525 HT, Nijmegen, The Netherlands

d) Child and Adolescent Psychiatry, Stockholm Health Care Services, Region Stockholm, Gävlegatan 22, 11330 Stockholm, Sweden

e) Curtin Autism Research Group, Essential Partner Autism CRC, School of Occupational Therapy, Social Work and Speech Pathology, Curtin University, Kent Street, Bentley, Perth, 6102, WA, Australia

*Correspondence: janina.neufeld@ki.se 


\section{Abstract}

Background. Synesthesia occurs more commonly in individuals diagnosed with Autism Spectrum Condition (ASC) and is associated with autistic traits and autism-related perceptual processing characteristics, including a more detail-focused attentional style and altered sensory sensitivity. Furthermore, autistic traits and autism-like sensory sensitivity show a synesthesia-dosage effect, since they increase with the amount of synesthesia types in synesthetes and with the degree of synesthesia (how consistently colors are associated with graphemes) in non-synesthetes.

Method. Here we investigated a predominantly non-synesthetic twin sample, enriched for ASC and other neurodevelopmental disorders ( $n=65,14-34$ years, 60\% female). We modelled the linear relationships between the degree of synesthesia and autistic traits, sensory sensitivity, and visual perception, both within-twin pairs (22 pairs) where all factors shared by twins are implicitly controlled (including $50-100 \%$ genetics), and across the entire cohort.

Results. Our results indicate that the degree of synesthesia is associated with autistic traits only within the attention to details domain and with sensory hyper-, but not hypo-sensitivity. These associations were stronger within-twin pairs compared to across the sample. Further, twins with a higher degree of synesthesia were better than their co-twins in identifying fragmented images in the Fragmented Pictures Test (FPT).

Conclusions. This study is the first twin study on the association between synesthesia and autism-related perceptual and clinical features, and the results suggest that a twin design can be more sensitive for detecting these associations. Consistent with previous findings, the results support an association between the degree of synesthesia and autism-related perceptual features, while utilizing a different self-report measure for sensory sensitivity. The novel finding of enhanced feature integration in the FPT in twins with a higher degree of synesthesia challenges the view of a generally more detail-focused attentional style in synesthesia and might be related to enhanced memory or mental imagery in more synesthetic individuals.

Key words: synesthesia, autism spectrum condition, sensory processing, perception, detail focus, twin study 


\section{Introduction}

Synesthesia is a non-pathological sensory condition where specific sensory inputs or concepts automatically trigger additional (often visual) sensations. For instance, a piano tone can trigger the sensation of an indigo blue sphere, moving in space. Synesthetic experiences can also be less complex: for instance, the number seven might lead to the sensation of lime green. Synesthesia is usually perceived consciously, occurs automatically, and is present from early childhood (Ward, 2013). Every synesthete has an individual pattern of associations which is remarkably consistent in adults, even over decades (Simner et al., 2005; Ward, 2013). Consistency is therefore one of the core criteria of synesthesia - which is usually assessed with a synesthesia consistency test (Baron-Cohen et al., 1996). Whether synesthesia is better described as a continuous trait in the population or as a binary condition is currently still debated. It is also possible that both views are meaningful (Ward, 2019), i.e., that some features of synesthesia follow a continuum while others are specific to suprathreshold synesthetes (i.e. individuals who both score within the synesthetic range on a consistency test and additionally perceive synesthesia consciously). Research on the genetics of synesthesia is still in its infancy, but early results indicate that it is a heterogenetic condition, involving genes associated with axonogenesis expressed during early childhood (Asher et al., 2009; A. Tilot et al., 2018; Tomson et al., 2011).

Both self-reported and objectively tested synesthesia are more common in individuals with Autism Spectrum Condition (ASC) (Baron-Cohen et al., 2013; Hughes et al., 2017; Neufeld et al., 2013). ASC is a neurodevelopmental entity characterized by difficulties in social communication and interaction, alongside restricted/repetitive patterns of behavior, interests, or activities and alterations in sensory perception (American Psychiatric Association, 2013). Synesthesia has also been suggested to play a key role for savant abilities, which occur in approximately $10 \%$ of individuals with ASC (Treffert, 2009). The co-occurrence between synesthesia and ASC seems to coincide with such talents (Baron-Cohen et al., 2007; Bouvet et al., 2014; Hughes et al., 2017), and single cases of savant synesthetes like Luria's S. described their synesthetic sensations as crucial for remembering or differentiating inputs (Luria \& Solotaroff, 1987). In addition, synesthetes seem to have an advantage in the acquisition of abilities resembling savant-talents (Hughes et al., 2019). Synesthesia is also associated with cognitive benefits in individuals without ASC, including enhanced memory, creativity and mental imagery (Ward and Simner, 2020). 
A link between synesthesia and ASC is further supported by studies showing that synesthetes have elevated autistic traits, and, similar to individuals with ASC (Ben-Sasson et al., 2009), an altered sensory processing style (Van Leeuwen et al., 2020; Van Leeuwen et al., 2019; Ward, et al., 2017a \& b). The latter includes both altered sensory sensitivity (hyperor hypo-sensitivity) and an increased attention to details. More specifically, synesthetes consistently show sensory hyper-sensitivity, based on both self-report (using the Glasgow Sensory Questionnaire; Robertson and Simmons, 2013) and on the Pattern Glare Test where Gabor patterns can evoke discomfort and sensory artefacts in more sensitive individuals (Van Leeuwen et al., 2019; Ward, et al., 2017a \& b). Of these three studies, two also found higher levels of self-reported sensory hypo-sensitivity in synesthetes (Ward, et al., 2017a \& b), but one did not (Van Leeuwen et al., 2019).

All three studies reported increased self-reported autistic traits, as assessed by the Autism Quotient (AQ; Baron-Cohen et al., 2001), in synesthetes. However, there was some inconsistency regarding which sub-domains where affected. The $A Q$ was originally constructed to cover five domains of autistic traits (Communication, Social skills, Imagination, Attention Switching and Attention to Details), with sub-scales showing varying degrees of internal consistency (Cronbach's alpha: .63-.77). However, factor analysis revealed a twofactor structure, with the Attention to Details domain being one factor and the remaining four sub-scales clustering together, referred to as AQ-Other here (Hoekstra et al., 2008). Ward et al. $(2017 a \& b)$ found that both the Attention to Details domain and the AQ-Other domain were significantly elevated in synesthetes compared to controls, but only scores on the Attention to Details domain resembled the autistic rather than the control sample's scores and showed a dosage effect, i.e., an increase in Attention to Details score correlated with the amount of experienced synesthesia types. Van Leeuwen et al (2019) found higher scores for synesthetes on the Social Skills sub-scale (within the AQ-Other domain) and the Attention to Details sub-scale, but a synesthesia dosage effect only with the Social Skills sub-scale. Finally, Burghoorn et al (2020) found the degree of grapheme-color synesthesia in nonsynesthetes, as indicated by synesthetic consistency scores, to correlate only with the total AQ score, but not the Attention to Details sub-scale (Burghoorn et al., 2020). Together, these self-report findings on autistic traits illustrate that elevated autistic traits in synesthetes span across both social/communication and perceptual/sensory domains.

Synesthetes have further been found to perform more accurate than controls on the Embedded Figures Test (EFT) and the Change Blindness Task (Van Leeuwen et al., 2019; Ward, et al., 2017a). Both these tasks require participants to suppress global information processing in order to detect smaller details, an ability that has been hypothesized and found to be enhanced in individuals with ASC (Mottron et al., 2006; Shah \& Frith, 1993). A recent 
meta-analysis nuances the idea of enhanced detail processing in autism, rather suggesting a somewhat slower processing of global information (Van der Hallen et al., 2015). The degree of grapheme-color synesthesia in non-synesthetes did however not correlate with EFT performance (Burghoorn et al. 2020). Synesthetes have also been found to have a higher motion coherence threshold than controls (Banissy et al., 2013; Van Leeuwen et al., 2019), i.e. needing a higher proportion of dots to move synchronously vs randomly in order to identify the global direction of movement. This indicates that they might have a reduced integration of features into a whole, similarly as previously found in people with ASC (Milne et al., 2002; Pellicano et al., 2005).

It is worth noting that synesthesia tends to co-occur with a broader range of mental conditions rather than being specifically linked to ASC, e.g. schizotypy, posttraumatic stress disorder, obsessive-compulsive disorder, or anxiety disorders (Carmichael et al., 2019; Hoffman et al., 2019; Tilot et al., 2019; Wendler and Schubert, 2019). Interestingly, altered sensory processing has been reported in several of these conditions, including obsessivecompulsive disorder or anxiety disorders, but received less attention than in ASC (Houghton et al., 2020).

Whether the association between synesthesia and autism-like perception is driven by familial factors such as genetics and upbringing is currently unknown. Twin and family studies have the power to address this question. Apart from two case reports of individual twin pairs discordant for synesthesia (Smilek, Dixon, and Merikle, 2005; Smilek et al., 2002), the only synesthesia twin group study published so far assessed concordance rates of colored sequence synesthesia in monozygotic $(M Z)$ vs dizygotic (DZ) twins, yielding suggestive evidence of both genetic and environmental influence on synesthesia (Bosley and Eagleman, 2015). A higher concordance rate in $M Z$ compared to $D Z$ twins suggested heritability, in line with studies indicating that synesthesia runs in families (Barnett et al., 2008; Baron-Cohen et al., 1996). Familial factors comprise both genetic and environmental aspects shared by family members. Twins share many environmental factors, such as parental age and health, socioeconomic background and many other aspects of their upbringing. In addition, MZ twins share all their genes, while DZ twins share on average $50 \%$ of their genes. In order to model the influences of genetics, shared, and non-shared environment quantitatively, large population-based twin or family samples are required. However, smaller twin samples can be used in order to investigate associations within-twin pairs, where the associations are not influenced by familial factors, given that these are shared between twins. This approach therefore allows investigating associations more directly, since familial factors that influence either the dependent or the independent variable can also modulate their association (strengthen or weaken it). As an example, exposure to colored letter sets in childhood, which 
has been shown to influence synesthetic color associations (Witthoft, Winawer, and Eagleman 2015; Witthoft and Winawer, 2006), is an environmental factor that is likely to be shared by twins (having a letter set toy at home or not). The current study is the first to investigate the link between synesthesia and autism-related characteristics in a twin sample, allowing to implicitly control for a wide range of familial confounding factors, and thereby investigating associations more directly and with higher sensitivity. In comparison, we also assess the same associations across the cohort, adjusting for twin clustering, and additionally including singletons (twins whose co-twin was not assessed). The latter analysis is more similar to regression analyses in non-twin samples and allows, in comparison to the analysis within-twin pairs, some inferences about the influence of familial factors.

Our study complements the existing literature methodologically in several further ways. First, we investigated an opportunistic sample that was not conditioned on the presence of synesthesia, but instead was clinically enriched for ASC and other neurodevelopmental disorders (NDDs). Second, we used a different measure to assess sensory sensitivity (Sensory Profile; Brown and Dunn, 2002), which has been used excessively within the autism literature (DuBois et al., 2017), but not yet in the context of synesthesia. Finally, in addition to the previously used Embedded Figures Test (EFT, Witkin, 1971), we also utilized the Fragmented Pictures Test (FPT, Kessler, Schaaf, and Mielke, 1993) as a test of detailoriented perception, which has not been used in previous synesthesia studies, assessing the ability to integrate fragments in order to construct a whole gestalt.

\section{Hypotheses}

We hypothesized that the degree of synesthesia (scoring more consistently, i.e. lower, on the synesthesia consistency test) would be associated with a) higher autistic traits b) increased sensory hyper- and hypo-sensitivity, and c) more detail-focused visual processing as indicated by faster disembedding in the EFT and the need for more visual information before being able to integrate fragments to a whole gestalt in the FPT.

\section{Methods}

\section{Participants}

Participants for this study were recruited from the Roots of Autism and ADHD Twin Study in Sweden (RATSS, Bölte et al. 2014). Within RATSS, individuals (twins) already completed a battery of different cognitive tests and questionnaires and were assessed diagnostically at an initial assessment. For the current study, 149 RATSS participants were assessed online for 
synesthesia, either directly after or up to eight years after the initial assessment within RATSS. The synesthesia assessment included a synesthesia consistency test and a questionnaire about different synesthesia types. Of the individuals who completed the synesthesia assessment, we excluded 66 individuals who did not complete the adult selfreport version of the $A Q$ (because they were below 16 years of age at the time of initial assessment) from analyses on sensory sensitivity and visual task performance. This because we wanted to assess all associations within the same cohort and because associations between measures assessed at different time points are more likely to be compromised in individuals who went through puberty between assessments. Of the remaining 83 individuals, 18 individuals did not have valid synesthesia consistency test scores due to a failure to follow task instructions (see section "Consistency test") so they were excluded from analyses (exclusion at step 2). The final included sample $(n=65)$ comprised 22 complete twin pairs and 21 singletons (twins whose co-twins either did not choose to take part in the online synesthesia assessment or were excluded due to an invalid consistency score). Sample characteristics for both the entire included sample and the 22 complete twin pairs are summarized in Table 1. Zygosity was determined based on a DNA test (Hannelius et al., 2007) for 18 of the complete twin pairs and a 4-item zygosity questionnaire for the four remaining twin pairs. Of the 22 complete twin pairs, 13 were monozygotic (MZ) and 9 dizygotic (DZ).

Twins in RATSS are predominantly recruited from a population twin sample (Anckarsäter et al., 2011), and selected if one or both twins show increased levels of autistic or ADHD traits based on a parent interview. As a consequence, the current sample was enriched for ASC and other NDDs. The final sample investigated in this study included 12 individuals with an NDD diagnosis (five of which had ASC; see Table 1). Written informed consent was obtained from all participants and/or their caregivers for participating in the RATSS study as a whole, and electronic informed consent was additionally collected online from all participants who also completed the synesthesia assessment. For completing the synesthesia online assessment, participants received a small compensation (worth 100 Swedish crowns; equivalent of $\sim 10$ U.S. dollars) in the form of a voucher that can be used in a wide range of shops. 
Table 1. Sample characteristics

\begin{tabular}{|l|cc|}
\hline & Full included sample & Complete twin pairs \\
\cline { 2 - 2 } N (female) & $65(39)$ & $44(29)$ \\
N NDD diagnosis (ASC) & $12(5)$ & $8(3)$ \\
MZ I DZ & $39 / 26$ & $26 / 18$ \\
Mean age (SD) at synesthesia & $26.7(4.6)$ & $26.8(4.8)$ \\
assessment & & $17-34$ \\
Age range at synesthesia assessment & $17-34$ & $23.9(5.4)$ \\
Mean age (SD) at first assessment & $23.2(5.4)$ & $16-32$ \\
Age range at first assessment & $14^{*}-32$ & $2.8(2.1)$ \\
Mean time passed between & $3.4(2.2)$ & $0-8$ \\
assessments in years (SD) & & \\
Range time passed between & $0-8$ & \\
assessments in years & & \\
\hline
\end{tabular}

Note. NDD = neurodevelopmental disorders; *one individual completed the adolescent/adult $A Q$ version (which is usually used form 16 years of age) despite being only 14 (almost 15) years old

\section{Synesthesia online assessment}

Screening questionnaire. Participants received a short description of synesthesia and then completed 15 multiple choice questions in which they were asked to rate to what extent they experienced different types of synesthesia. The questions contained examples of possible synesthetic experiences. Answer possibilities were: "yes", "yes, to some extent", "no" and "I don't know". In addition, an open question concerning potential synesthesia types that had not been addressed was included. The first two multiple choice questions concerned lettercolor, and digit-color synesthesia, and the answers to these questions, in combination with the consistency score, were used to identify grapheme-color synesthetes.

Consistency test. Participants completed a Swedish version of an online grapheme-color consistency test, which had been used previously as a Dutch version (Van Leeuwen et al., 2019; Cuskley et al. 2019). This test is similar to an online test (Eagleman et al., 2007) that differentiates between synesthetes and non-synesthetes with a high $(>80 \%)$ sensitivity and specificity (Rothen et al., 2013). Stimuli were the 26 letters of the Latin alphabet plus the

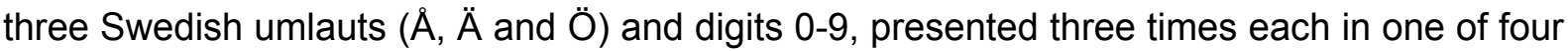
pseudorandom orders (avoiding an item to occur twice in a row) assigned randomly to 
participants. Participants were presented with one stimulus at a time and asked to choose the color that fit the stimulus best by clicking in a color matrix (see Supplementary Figure 1). Synesthetes tend to choose the exact same color on each presentation of the same stimulus, allowing the test to discriminate between synesthetes and non-synesthetes, who choose color associations less consistently. For each participant, we calculated a consistency score using Euclidean distances in CIELUV color space between participant responses for the three different trials per item (Rothen et al. 2013), with lower consistency scores indicate higher levels of synesthetic color consistency (= similar colors chosen). Only items where colors were chosen for all three trials were included. The consistency scores of 18 participants were deemed invalid because they had less than five valid items or only chose one or two different colors across all items (see Supplementary Material for details). Identification of synesthetes. Participants were categorized in grapheme-color synesthetes and non-synesthetes based on their consistency test results and their answers on the synesthesia questionnaire. Participants having a valid consistency score and scoring below a cut-off value of 135.30 for digits, letters, or both categories were classified as synesthetes if they in addition also reported experiencing grapheme-color synesthesia for the relevant category in the questionnaire (answering "yes" or "yes, to some extent" to one or both of the first two questions). Participants were included in the analyses regardless whether they qualified as synesthetes or not.

\section{Measures acquired during the initial assessment}

In their initial assessment within the RATSS study, twins were assessed diagnostically and completed a large battery of questionnaires and tests during a 2.5 days lab visit. Details are described below.

Diagnostic assessment. Twins were assessed by a team of experienced clinicians. Diagnosis for ASC or other NDDs such as ADHD, tic disorders, specific learning disorders and intellectual disability (ID), as well as psychiatric disorders such as depression and anxiety were determined based on a multitude of information sources. These included medical history, parent interviews and gold standard diagnostic tools (First \& Gibbon, 2004; Kaufman et al., 1997; Kooij, 2010; Lord et al., 2012, Lord, Rutter, and Couteur, 1994).

Autistic traits. Autistic traits were measured by the Swedish adult self-report version of the Autism Quotient, AQ (Baron-Cohen et al., 2001, https://www.autismresearchcentre.com/ arc_tests). Sub-scores were calculated for the two sub-domains "Attention to Details" and "AQ-Other" according to previous factor analyses (Hoekstra et al., 2008). 
Sensory processing. Variation in sensory processing was assessed with the self-report adolescent/adult Sensory Profile (AASP), a questionnaire that comprises 60 questions dividing into four quadrants (15 questions each), related to sensory hyper- or hyporesponsiveness across different sensory modalities (Brown and Dunn, 2002). The Sensory Profile is the most commonly used measure of sensory hyper- and hypo-responsiveness in studies involving clinical samples such as adults and adolescents with ASC (DuBois et al., 2017), but has so far not been used in synesthesia studies. The quadrants Sensory Sensitivity and Low Registration represent higher or lower sensory thresholds (hyper- and hypo-sensitivity). The remaining two AASP quadrants, Sensation Seeking and Sensation Avoiding, represent actions undertaken to limit or increase sensory input. We only included the quadrants representing high vs low sensory thresholds in our analyses (Sensory Sensitivity and Low Registration), because we believe that they represent purer measures of hyper- and hypo-responsiveness (with less impact of other factors such as self-regulation abilities) and because we wanted to avoid including several correlated predictor variables within the same model (correlation between the different AASP quadrants in our sample was $r=-.34$ to .68$)$.

Perceptual tasks. Two tasks (pen and paper versions) were used to assess the participants' style of global vs local visual information processing (see Supplementary Figure 2 for an example). The Embedded Figures Task (EFT; adult version) requires dis-embedding different smaller targets from 12 larger distracting contexts (one target per context) within 180 seconds (Witkin, 1971). For each of the 12 contexts, the amount of errors (pointing to the wrong area) and the time until correct response were noted. The maximum time of 180 seconds was noted if the participant was unable to correctly identify the target (overall maximum time $=2160$ seconds). Mean reaction time till correction response and the amount of errors were used as the outcome measures of this task.

The Fragmented Pictures Task (FPT) assesses the ability to integrate visual fragments into a meaningful whole (Kessler et al., 1993). Participants browsed with a steady pace through 10 sets of fragmented drawings of objects, animals or persons that were gradually completed in 10 sequential steps, and were asked to identify each drawing verbally as soon as possible. The latter requires both bottom-up visual feature integrations, and a top-down matching between image and internal shape representations from memory (Van Eylen et al., 2015). The number of images (corresponding to state of completeness) needed in order to correctly identify each drawing was summed up across the 10 trials as the total FPT score. 
Four of the 65 included participants were lacking EFT test results of which one was additionally lacking FPT results, leading to a total of 61 participants (20 complete twin pairs) included in the analyses.

Intellectual ability. The Wechsler Intelligence Scales for Children (if below 18 years of age) or Adults, fourth editions (WISC-IV/WAIS-IV) were used to assess intellectual ability (Wechsler, 2003 \& 2008). The General Ability Index (GAI) was calculated as measure of IQ, based on three verbal comprehension and three perceptual reasoning subtests.

\section{Statistical analysis}

General performance assessment. We report all the mean scores and values obtained from the whole sample included in this study, and we assessed whether these scores and values were extremely different compared to previously published scores and values from typically developing participants (TD). We performed this check to evaluate the representativeness of our clinically enriched cohort compared to studies including TD participants and to verify that questionnaires and tasks were completed according to instructions. Furthermore, we explored the data set by comparing the group means of dependent and independent variables between individuals with and without any NDD diagnoses, and between individuals that were included in the study and those that were excluded at step 2 because they did not reach a valid consistency score (see Supplementary Tables 1 and 2).

Main modeling analyses. Three conditional linear regression analyses were conducted in $\mathrm{R}$ within the Generalized Estimating Equations (gee) framework with doubly robust standard errors (drgee package) (Zetterqvist and Sjölander, 2015), in order to investigate within pair associations between synesthesia and autistic traits, sensory sensitivity and visual test performance. More specifically, each of the 22 twin pairs (or in case of the visual tests 20 pairs) formed a cluster and the synesthetic consistency score was the dependent variable in all three models while either autistic traits (AQ-Attention to Details and AQ-Other), sensory sensitivity (Sensory Sensitivity and Low Registration) or visual task performance (EFT reaction time and error rate, and the total FPT score) were the main independent variables.

In a second step, three linear regressions with the same variables were conducted across the full cohort, were each participant was treated as individual but cluster-robust standard errors were estimated in order to account for the non-independence of twins. This second analyses resembles therefore a conventional linear regression and is hence more comparable with previous studies on non-twin samples. 
Standardized scores (mean-centered values divided by the standard deviation) of all measures were used, leading to standardized model estimates which can be interpreted as effect size estimates. In all models, we controlled for the presence of an NDD diagnosis $(0=$ no diagnosis, 1 = diagnosed with ASC, ADHD, specific learning disorder and/or tic disorders) and age when completing the synesthesia consistency test, since our sample was clinically enriched with a wide age range. We also included general intellectual ability (GAI, at the time of the initial assessment in RATSS), to control for IQ differences and increase sensitivity. We additionally speculated that synesthesia consistency scores might be affected by IQ, e.g. because individuals with higher IQ might remember more often which colors they chose for previous items, or be better at finding strategies to remember the colors. However, this latter hypothesis has not been tested previously, to the best of our knowledge.

\section{Results}

\section{General performance assessment}

For an overview of means and standard deviations of all included variables across the whole group and for complete twin pairs, please see Table 2.

AQ scores were within the expected range for a sample containing predominantly individuals without an ASC diagnosis, reaching a mean total $A Q$ score of $14.0(S D=8.9)$ which is within the reported range for non-clinical populations (range: $11.9-17.6 ; \mathrm{Cl}=13.0-17.1$, Table 2 from a meta-analysis by Ruzich et al., 2015). Sensory Profile scores in the current sample were comparable to scores from earlier representative studies using the Adult Sensory Profile in the general population (Kamath et al., 2020; Brown et al., 2003). For Sensory Sensitivity, the mean score of 32.1 (SD = 9.9) is similar to the mean score in Kamath et al., $2020(30.77 \pm S D=6.29)$ and slightly below one SD from the mean score $(38.0 \pm S D=5.4)$ in Brown et al. (2003). For Low Registration, the mean score of 27.7 (SD = 8.4) is comparable to the scores from both earlier studies $(28.14 \pm \mathrm{SD}=4.48$, Kamath et al., 2020; $31.1 \pm \mathrm{SD}=4.9$, Brown et al., 2003).

The performance on the visual tests in this sample was within the expected range. On the FPT, participants identified the images between step 6 and 7 on average (total score across 10 trials of $64.5, S D=5.6)$, similar to comparable fragmented pictures studies reporting identification at around 60\% visibility levels (e.g. Snodgrass and Corwin, 1988; Doniger et al., 2001). EFT performance (RT $46.2 \mathrm{~s}, \mathrm{SD}=22.7$ ) in our sample was similar to the reaction times of normative individuals (51.5 \pm SD $=12.6$, Table 3 from Bölte et al., 2007). 
Intellectual ability in the included sample was slightly higher (mean $=105.9, S D=14.2)$ than average in the general population.

For further details on performance see the Supplementary Materials where we discuss and list (Supplementary Table 1 and 2) additional descriptive statistics of data from participants excluded at step 2 and participants with and without an NDD diagnosis.

Table 2. Descriptive Results

\begin{tabular}{|l|cc|}
\hline & Full included sample & Complete twin pairs \\
\cline { 2 - 2 } Mean (SD) consistency score & $241.8(89.0)$ & $240.94(82.01)$ \\
N verified synesthetes & 4 & 4 \\
Mean number of self-reported & $1.6(1.4)$ & $1.4(1.4)$ \\
synesthesia types (SD)* & & \\
Mean (SD) IQ & $105.9(14.2)$ & $106.6(14.5)$ \\
Mean (SD) AQ total & $14.0(8.9)$ & $13.0(7.9)$ \\
Mean (SD) AQ-Attention to Details & $3.8(2.1)$ & $3.9(2.1)$ \\
Mean (SD) AQ-Other & $10.0(7.6)$ & $9.3(6.7)$ \\
Mean (SD) Sensory Sensitivity & $32.1(9.9)$ & $31.3(8.6)$ \\
Mean (SD) Low Registration & $27.7(8.4)$ & $27.0(7.7)$ \\
Mean (SD) EFT RT (s) & $46.2(22.7)$ & $46.2(21.9)$ \\
Mean (SD) EFT errors & $4.5(3.6)$ & $4.7(3.8)$ \\
Mean (SD) FPT total score & $64.5(5.6)$ & $64.2(5.1)$ \\
\hline
\end{tabular}

Note. ${ }^{*}$ The mean number of synesthesia types was calculated from the 16 items of the questionnaire, where types that cluster together according to Novich et al. (2011) were only counted as one, hence the maximal number of self-reported synesthesia types was six.

Regression outcomes

Regression analyses findings are described below per model, each calculated both withintwin pairs and across individuals, and all models' results are summarized in Table 3.

Degree of synesthesia and autistic traits. Within-twin pairs, only Attention to Details, but not the $A Q$-Other subscale of the $A Q$, predicted higher synesthetic consistency (= lower consistency scores) within-twin pairs, $(\beta=-.66,95 \% \mathrm{Cl}=-1.21,-.11, p=.019)$. This means that twins with a one standard deviation higher Attention to Details score compared to their co-twins would score about half a standard deviation lower in the consistency test, i.e., more 
consistent. Across individuals, an effect in the same direction was not significant $(\beta=-.24$, $95 \% \mathrm{Cl}=-.54, .03, p=.087)$.

Degree of synesthesia and sensory sensitivity. Hyper-, but not hyposensitivity, predicted stronger synesthetic consistency, in line with our hypothesis, both within pairs ( $\beta=-.60,95 \%$ $\mathrm{Cl}=-1.14,-.06, p=.031)$ and across individuals $(\beta=-.35,95 \% \mathrm{Cl}=-0.68,-.00, p=.044)$.

Degree of synesthesia and perceptual task performance. Dis-embedding ability, as assessed with the EFT (reaction times and number of errors), was not associated with the degree of synesthetic consistency, neither within-twin pairs (RT: $\beta=.17,95 \% \mathrm{Cl}=-.56, .90, p=.652$; accuracy: $\beta=-.40,95 \% \mathrm{Cl}=-.91, .11, p=.121)$, nor across individuals (RT: $\beta=.11,95 \% \mathrm{Cl}$ $=-.56, .90, p=.538$; accuracy: $\beta=-.03,95 \% \mathrm{Cl}=-.91, .11, p=.886)$.

We found no evidence for our hypothesis that people who perform worse on the FPT would score more like a synesthete on the consistency test. Instead, better performance (i.e. a reduced need of visual information for correct object identification) in the FPT predicted higher synesthetic consistency (a lower score) within-twin pairs $(\beta=.47,95 \% \mathrm{Cl}=.08, .85, p$ $=.017)$. No association was observed across individuals $(\beta=.17,95 \% \mathrm{Cl}=-.14, .48, p=$ .274).

Effects of controlling variables: IQ, NDD diagnosis and age. Intellectual ability was not associated with the degree of synesthesia within-twin pairs in any model ( $\beta$-range: -.10 to .04 , p-range .089 to .799). Across individuals, higher $I Q$ consistently predicted higher synesthetic consistency, as strongest included predictor ( $\beta$-range: -.03 to $-.04, p$-range: .030 to <.001). NDD diagnosis and age were not associated with synesthetic consistency in this sample (please see Table 3 for details). 
Table 3. Regression results

\begin{tabular}{|c|c|c|c|c|c|c|}
\hline & \multicolumn{3}{|c|}{ Within-twin pairs $(n=44)$} & \multicolumn{3}{|c|}{ Across individuals $(n=65)$} \\
\hline & $\beta(95 \% \mathrm{Cl})$ & SE & $p$ & $\beta(C I)$ & $S E$ & $p$ \\
\hline \multicolumn{7}{|c|}{ Model 1: Autistic traits } \\
\hline Attention to Details & $-.66(-1.21,-.11)$ & .28 & .019 & $-.24(-.51, .03)$ & .14 & .087 \\
\hline AQ-Other & $.04(-.51, .60)$ & .28 & .878 & $.07(-.19, .33)$ & .13 & .592 \\
\hline GAI (scaled score) & $-.40(-.85, .06)$ & .23 & .089 & $-.42(-.64,-.22)$ & .01 & $<.001$ \\
\hline NDD diagnosis & $-.12(-1.57,1.33)$ & .16 & .870 & $-.09(-.77, .60)$ & .35 & .809 \\
\hline Age & - & - & - & $.02(-.04, .07)$ & .03 & .575 \\
\hline \multicolumn{7}{|c|}{ Model 2: Sensory Profile } \\
\hline Sensory Sensitivity & $-.60(-1.14,-.06)$ & .28 & .031 & $-.35(-0.68,-.01)$ & .17 & .044 \\
\hline Low Registration & $-.48(1.17, .22)$ & .36 & .179 & $.26(-.09, .60)$ & .17 & .149 \\
\hline GAI (scaled score) & $-.30(-.75, .15)$ & .23 & .192 & $-.42(-.63,-.21)$ & .11 & $<.001$ \\
\hline NDD diagnosis & $.25(-1.39,1.90)$ & .84 & .763 & $-.15(-.70, .40)$ & .28 & .595 \\
\hline Age & - & - & - & $.02(-.03, .07)$ & .03 & .470 \\
\hline \multicolumn{7}{|c|}{ Model 3: Perceptual task performance } \\
\hline EFT (accuracy) & $-.40(-.91, .11)$ & .26 & .121 & $-.03(-.41, .35)$ & .19 & .886 \\
\hline EFT (RT) & $.17(-.56, .90)$ & .37 & .652 & $.11(-.24, .45)$ & .18 & .538 \\
\hline FPT score & $.47(.08, .85)$ & .20 & .017 & $.17(-.14, .48)$ & .16 & .274 \\
\hline GAI (scaled score) & $.10(-.64, .83)$ & .38 & .799 & $-.28(-.53,-.03)$ & .13 & .030 \\
\hline NDD diagnosis & $-1.64(-3.37, .08)$ & .88 & .062 & $.20(-.78, .38)$ & .30 & .501 \\
\hline Age & - & - & - & $.01(-.05, .06)$ & .03 & .838 \\
\hline
\end{tabular}

Note. Regression outcomes (with 2-sided p-values) from conditional linear regressions within-twin pairs (left column) and linear regressions across individuals with standard errors adjusted for twin clustering (right column). $A Q=$ Autism Quotient, GAl = General Ability Index, $N D D=$ Neurodevelopmental Disorder, EFT = Embedded Figures Test, FPT = Fragmented Picture Test, $\beta=$ standardized regression coefficient, $\mathrm{Cl}=$ confidence interval, $S E=$ standard error. 


\section{Discussion}

In this study, we investigated the relationships between the degree of synesthesia (synesthetic consistency) and autistic traits, sensory sensitivity and detail focus in visual perception in a twin sample. We controlled for NDD diagnosis, general intellectual ability and across individuals also for age (which is implicitly controlled for within-twin pairs). Importantly, shared environmental factors, such as family background, and $50-100 \%$ of genetic effects are additionally implicitly controlled for within-twin pairs, and hence the within-pair associations are not influenced by these familial factors. Four main findings emerged. First, only Attention to Details, but not the other domain of autistic traits (including sub-scales related to social skills), predicted higher synesthetic consistency within-twin pairs. Second, sensory hyper-sensitivity but not hypo-sensitivity predicted higher synesthetic consistency. Third, these within-twin pair associations pointed in the same direction as associations across all individuals, but were stronger within pairs, indicating that the within-pair analysis was more sensitive in order to detect these effects. Finally, the need for visual information in the FPT (but not dis-embedding ability in the EFT) was associated with synesthetic consistency within-twin pairs. Twins who needed less visual information than their co-twins in order to construct a meaningful whole from a fragmented image scored more like a synesthete on the consistency task. The latter finding was contrary to our hypothesis that individuals who are more consistent in the synesthesia test would integrate visual fragments less automatically into a whole gestalt because of a more detail-focused attentional style.

\section{Autistic traits and the degree of synesthesia}

Similar to previous studies and in line with our hypothesis, we found a positive association between the degree of synesthesia and autistic traits. We found this association to be specific to the area of Attention to Details. This association was significant within-twin pairs but only a trend in the same direction across all individuals. Our results are consistent with prior work that found increased autistic traits in synesthetes compared to non-synesthetes, with a stronger increase within the Attention to Details compared to the AQ-Other domain (Ward et al. 2017a, b). That work also found the amount of different synesthesia types in synesthetes to correlate with Attention to Detail but not with the AQ-Other sub-domain. In Van Leeuwen et al. (2019), synesthetes similarly showed an increase in Attention to Detail compared to non-synesthetes, although this was also the case for one of the AQ-Other subscales, namely Social Skills.

In contrast to the previously mentioned studies, we did not compare synesthetes with non-synesthetes but instead used the degree of synesthesia as assessed 
as continuous synesthetic consistency score in an opportunistic sample. Using the same approach, Burghoorn et al. (2020) found a correlation between degree of synesthesia and autistic traits, but only for the total $A Q$ score and not the Attention to Detail sub-domain. In contrast to Burghoorn et al. (2020), we investigated a clinically enriched twin sample (where $18 \%$ had an NDD diagnosis), used a different AQ-scoring (according to the original $A Q$, validated in a British sample), and we adjusted for IQ, age and NDD diagnosis. These differences can possibly explain the discrepancy in the results - checking in our data, a regression analysis with the total $\mathrm{AQ}$ score revealed no significant associations (see Supplementary Text). Our results confirm the finding of Burghoorn et al. (2020) that an association between the degree of synesthesia and autistic traits is also apparent in a nonsynesthete sample (our main regression results are similar after exclusion of the four verified synesthetes or twin pairs where at least one was identified as a synesthete, respectively, and hold for the $A Q$ attention to details sub-scale and the FPT, see Supplementary Table 3). Altogether, in line with previous evidence, our findings support the notion that Attention to Detail is more consistently associated with synesthesia than autistic traits that are more closely related to social functioning. This is consistent with the idea that the link between synesthesia and autism is not a general one, but specifically at the level of perceptual and attentional processes.

\section{Sensory sensitivity and degree of synesthesia}

Partially confirming our hypothesis, we found an association between sensory hypersensitivity (assessed with the Sensory Sensitivity subscale of the AASP) and degree of synesthesia. This association was robust both within-twin pairs and across the entire sample. Increased sensory sensitivity predicted a higher degree of synesthesia (lower consistency scores). In contrast, contrary to our hypothesis, we did not find such an association with hypo-sensitivity (assessed with the Low Registration sub-scale of the AASP). These results correspond to findings by Van Leeuwen et al (2019) that synesthetes score higher than controls on sensory hyper-sensitivity, but not hypo-sensitivity, on the Glasgow Sensory Questionnaire (GSQ). However, Ward at al. (2017a\&b) found elevated scores in both hyperand hypo-sensitivity GSQ domains. In one of these studies, hyper-sensitivity in synesthetes was additionally indicated by more self-reported discomfort evoked by Gabor patterns in the Pattern glare test (Ward, et al., 2017b). Taken together, the association between synesthesia and hypo-sensitivity remains controversial, while the association between synesthesia and hyper-sensitivity occurred more consistently across several studies. Our study adds to this 
evidence by using a different self-report measure that is widely used in the autism literature (AASP instead of the GSQ).

\section{Visual perception and degree of synesthesia}

Previous studies found that synesthetes performed more accurately in different versions of the EFT (Van Leeuwen et al., 2019; Ward, 2017a), in line with a more detail-oriented visual performance style resembling the perceptual style commonly found in individuals diagnosed with ASC (Shah \& Frith, 1993, Ben-Sasson et al., 2009). Contrary to these studies and our hypothesis, we did not find an association between the degree of synesthesia and disembedding ability while using a different version of the task. While we used the original onpaper version of the EFT (Witkin et al., 1971), the two previous studies used online computer versions of the task. The on-paper version of the test has the advantage that we can be more confident of participants' attentional engagement, and the disadvantage that the interaction with the test administrator adds a social component, which could affect the performance in some individuals more than in others. Van Leeuwen et al (2019) used a more recent version of the task (Leuven Embedded Figures Test; L-EFT, De-Wit et al., 2007) that allows differentiating different degrees of dis-embedding difficulty. They only found convincing group differences between synesthete and non-synesthete participants in the most difficult condition. Unfortunately, the test version we used does not allow differentiating different degrees of difficulty: however, given the maximum time for completion of one trial (180 s) we assume our version is quite hard to complete (RTs in Van Leeuwen et al., 2019 in the most difficult condition were $8 \mathrm{~s}$ on average). Further, accuracy is measured differently in our version of the EFT, where several errors could be made per trial, compared to the L-EFT, where each trial is counted as either correct or incorrect, hence we cannot compare error rates. Further, performance in the EFT in people diagnosed with ASC is usually modulated by general intellectual ability (Muth, Hönekopp, and Falter, 2014) and was also correlated with GAl in our study ( $r=.34$ for accuracy and $r=.38$ for reaction time), which might have impacted the results. On the other hand, Burghoorn et al (2020) also did not find an association between L-EFT performance and degree of synesthesia, which is in line with our study. They concluded that better L-EFT performance might be specific to supra-threshold synesthesia, which might also explain our null result.

In contrast to our prediction, we found that twins who needed less visual information than their co-twins in the FPT scored more synesthetically. We had predicted the opposite because a more detail-focused attention style has been hypothesized to not only be associated with advantages in detecting details, but also a reduced drive to integrate details 
into a more global picture (Shah \& Frith, 1993). Further, previous studies found reduced feature integration in synesthetes, as indicated by the need for more dots to move in the same direction in order to detect coherent motion (Banissy et al., 2013; Van Leeuwen et al., 2019). However, Burghoorn et al. (2020) did not find an association between motion coherence thresholds and the degree of synesthesia. While both the FPT and the motion coherence task require the integration of visual features, the tasks differ in many ways. As an example, FPT performance is partly dependent on matching the image with representations from memory. Since synesthetes have previously been shown to outperform nonsynesthetes in a wide range of memory tasks (Ward et al., 2019), a better FPT performance in more synesthetic individuals might be driven by memory effects. Further, synesthetes seem to have more vivid visual mental imagery (Ward and Simner, 2020), which likely is also required in the FPT matching process. It remains to be investigated how FPT performance relates to supra-threshold synesthesia. Finally, studies in individuals with autism found evidence for more locally biased attention to be more consistent if the task leaves it to participants to attend either the global picture or smaller details (Van der Hallen et al., 2015) but not when explicitly instructed to focus on global features (Mottron et al., 2003). In a similar way, synesthetes might overcome their more detail-oriented default attentional style in order to solve the task.

\section{Covariate effects}

In this study, we did not find an association between NDD diagnosis and degree of synesthesia. Further, of the four individuals identified as synesthetes, none had an NDD diagnosis. Since our NDD sample was small and heterogeneous, these results need to be interpreted with caution, but they might indicate the link between synesthesia and ASC does not generalize to a link between synesthesia and NDD. Further, it seems that individuals with NDD diagnoses or lower IQ were less likely to take part in this study and complete the online test correctly (please see Supplementary Text and Supplementary Table 1 and 2). Our clinically enriched sample did however have the advantage that individuals with more extreme trait levels were included. Future studies should investigate the degree of synesthesia and its prevalence in larger NDD samples and in a wider range of psychiatric trait measures, with special attention to the altered sensory processing that seems to be a common thread, across diagnostic boundaries. Intellectual ability was also associated with higher synesthetic consistency across individuals. The latter might either indicate that higher $\mathrm{IQ}$ increases the likelihood of achieving more consistent scores, or that more synesthetic individuals tend to have a higher IQ. Future studies should investigate this question in a larger typically developed sample with IQ as main predictor. 
Within-twin pairs, all factors shared by twins are implicitly adjusted for. An environmental factor that might influence an individual's synesthetic consistency and which is more likely to be shared by twins compared to unrelated individuals is the exposure to colored letter sets in childhood, which influences synesthetic color associations (Witthoft et al., 2015; Witthoft and Winawer, 2006). In addition, factors that might have added noise to the model across individuals might be shared by twins, hence potentially weakening the across sample associations but not the within pair effects. Stronger associations within-twin pairs compared to across the sample in this study suggest an increased sensitivity within pairs. In this study, one such factor might have been the time that had passed between initial and online assessment, which was the same for twins of a pair. Interestingly, the association between cognitive ability and synesthetic consistency was only significant across individuals, but lost within pairs. This association was strong across the sample despite the time gap between assessments, but might be driven by shared familial factors, for instance genetics.

\section{Strengths and limitations}

Strengths of our approach are the more sensitive twin design and the clinically enriched sample. We also used novel ways of assessing sensory sensitivity (the Sensory Profile) and perceptual processing (the Fragmented Pictures Test) compared to earlier synesthesia studies, allowing us to confirm as well as expand on previous findings. Limitations of our design are the limited sample size for regression analyses $(n=65)$, although the sample size is still larger than in the earlier paper by Burghoorn et al. (2020). Also, our sample did not consist solely of complete twin pairs but also included singletons in analyses across individuals, and hence, the samples were not entirely identical within-twin pairs and across the sample. Finally, an additional limitation is the time gap between the initial assessment in RATSS (perceptual tests, questionnaires) and the synesthesia experiment, which could be as long as 8 years ( $\sim 3$ years on average). While it is rather unlikely that the time gap has introduced associations that otherwise would not have been found, it is quite likely that it introduced some noise, which might have weakened the associations.

\section{Conclusion}

The current study complements previous evidence for a link between synesthesia and autistic traits (in the perceptual domain) and sensory hyper-sensitivity, using several novel questionnaires and tests compared to previous research, and a clinically enriched twin 
sample. The findings are in line with the idea that the link between autism and synesthesia is strongest at the level of perceptual and attentional processes. Importantly, all associations were more pronounced within-twin pairs, where they are more direct because a large amount of familial factors are implicitly controlled. Results from visual tests were less consistent with the literature and demand to be investigated in larger samples including more suprathreshold synesthetes.

\section{Acknowledgements}

We acknowledge The Swedish Twin Registry, which is managed by Karolinska Institutet and receives funding through the Swedish Research Council under the grant no 2017-00641, for access to data. We would also like to thank all participants of the RATSS project as well as our colleagues at the KIND center for their valuable contributions. The study was supported by Riksbankens Jubileumsfond (no. P18-0817:1). The RATSS project was supported by the Swedish Research Council (no. 2016-01168), "Forskningsrådet för miljö, areella näingar och samhällsbyggande" (FORMAS; no. 259-2012-24), The Swedish Brain Foundation (Hjärnfonden; no. FO2014-0228 \& no. FO2018-0053), Region Stockholm (SLL's Anslag till forskning, utveckling och utbildning "ALF medicin"; no. 20140134 \& no. 20170016) and Innovative Medical Initiatives (IMI) (no. 115300; EU-AIMS (2012-2017).

\section{Competing interests statement}

S. Bölte discloses that he has in the last three years acted as an author, consultant or lecturer for Shire, Medice, Roche, Eli Lilly, Prima Psychiatry, GLGroup, System Analytic, Kompetento, Expo Medica, Prophase, and receives oyalties for text books or diagnostic instruments from Huber/Hogrefe, Kohlhammer and UTB. The remaining authors declare that they have no conflicts of interest.

\section{References}

American Psychiatric Association. (2013). Diagnostic and statistical manual of mental disorders, (DSM-5®). American Psychiatric Pub.

Anckarsäter, H., Lundström, S., Kollberg, L., Kerekes, N., Palm, C., Carlström, E., Långström, N., Magnusson, P. K., Halldner, L., \& Bölte, S. (2011). The child and 
adolescent twin study in Sweden (CATSS). Twin Research and Human Genetics, 14(06), 495-508.

Asher, J. E., Lamb, J. A., Brocklebank, D., Cazier, J.-B., Maestrini, E., Addis, L., Sen, M., Baron-Cohen, S., \& Monaco, A. P. (2009). A whole-genome scan and fine-mapping linkage study of auditory-visual synesthesia reveals evidence of linkage to chromosomes 2q24, 5q33, 6p12, and 12p12. The American Journal of Human Genetics, 84(2), 279-285.

Banissy, M. J., Tester, V., Muggleton, N. G., Janik, A. B., Davenport, A., Franklin, A., Walsh, V., \& Ward, J. (2013). Synesthesia for color is linked to improved color perception but reduced motion perception. Psychological Science, 24(12), 2390-2397.

Barnett, K. J., Finucane, C., Asher, J. E., Bargary, G., Corvin, A. P., Newell, F. N., \& Mitchell, K. J. (2008). Familial patterns and the origins of individual differences in synaesthesia. Cognition, 106(2), 871-893.

Baron-Cohen, S., Bor, D., Billington, J., Asher, J., Wheelwright, S., \& Ashwin, C. (2007). Savant memory in a man with colour form-number synaesthesia and asperger. Journal of Consciousness Studies, 14(9-1), 237-251.

Baron-Cohen, S., Burt, L., Smith-Laittan, F., Harrison, J., \& Bolton, P. (1996). Synaesthesia: Prevalence and familiality. Perception, 25(9), 1073-1079.

Baron-Cohen, S., Johnson, D., Asher, J., Wheelwright, S., Fisher, S. E., Gregersen, P. K., \& Allison, C. (2013). Is synaesthesia more common in autism? Molecular Autism, 4(1), 40.

Baron-Cohen, S., Wheelwright, S., Skinner, R., Martin, J., \& Clubley, E. (2001). The autismspectrum quotient $(A Q)$ : Evidence from asperger syndrome/high-functioning autism, malesand females, scientists and mathematicians. Journal of Autism and Developmental Disorders, 31(1), 5-17.

Ben-Sasson, A., Hen, L., Fluss, R., Cermak, S. A., Engel-Yeger, B., \& Gal, E. (2009). A meta-analysis of sensory modulation symptoms in individuals with autism spectrum disorders. Journal of Autism and Developmental Disorders, 39(1), 1-11.

Bölte, S., Holtmann, M., Poustka, F., Scheurich, A., \& Schmidt, L. (2007). Gestalt perception and local-global processing in high-functioning autism. Journal of Autism and Developmental Disorders, 37(8), 1493-1504. 
Bölte, S., Willfors, C., Berggren, S., Norberg, J., Poltrago, L., Mevel, K., Coco, C., Fransson, P., Borg, J., \& Sitnikov, R. (2014). The roots of autism and ADHD twin study in Sweden (RATSS). Twin Research and Human Genetics, 17(03), 164-176.

Bosley, H. G., \& Eagleman, D. M. (2015). Synesthesia in twins: Incomplete concordance in monozygotes suggests extragenic factors. Behavioural Brain Research, 286, 93-96.

Bouvet, L., Donnadieu, S., Valdois, S., Caron, C., Dawson, M., \& Mottron, L. (2014). Veridical mapping in savant abilities, absolute pitch, and synesthesia: An autism case study. Frontiers in Psychology, 5.

Brown, C., \& Dunn, W. (2002). Adolescent/Adult Sensory Profile User's Manual. Pearson.

Brown, C., Cromwell, R. L., Filion, D., Dunn, W., \& Tollefson, N. (2003). Sensory processing in schizophrenia: Missing and avoiding information. Schizophrenia research, 55(1-2), 187-195.

Burghoorn, F., Dingemanse, M., van Lier, R., \& van Leeuwen, T. M. (2020). The Relation Between Autistic Traits, the Degree of Synaesthesia, and Local/Global Visual Perception. Journal of Autism and Developmental Disorders. https://doi.org/10.1007/s10803-019-04222-7

Carmichael, D. A., Smees, R., Shillcock, R. C., \& Simner, J. (2019). Is there a burden attached to synaesthesia? Health screening of synaesthetes in the general population. British Journal of Psychology (London, England: 1953), 110(3), 530-548.

Cuskley, C., Dingemanse, M., Kirby, S., \& Van Leeuwen, T. M. (2019). Cross-modal associations and synesthesia: Categorical perception and structure in vowel-color mappings in a large online sample. Behavior research methods, 51(4), 1651-1675.

De-Wit, L., Huygelier, H., Van der Hallen, R., Chamberlain, R., \& Wagemans, J. (2017). Developing the Leuven Embedded Figures Test (L-EFT): testing the stimulus features that influence embedding. PeerJ, 5, e2862.

Doniger, G. M., Foxe, J. J., Schroeder, C. E., Murray, M. M., Higgins, B. A., \& Javitt, D. C. (2001). Visual perceptual learning in human object recognition areas: a repetition priming study using high-density electrical mapping. Neuroimage, 13(2), 305-313. 
DuBois, D., Lymer, E., Gibson, B., Desarkar, P., \& Nalder, E. (2017). Assessing sensory processing dysfunction in adults and adolescents with autism spectrum disorder: A scoping review. Brain Sciences, 7(8), 108.

Eagleman, D. M., Kagan, A. D., Nelson, S. S., Sagaram, D., \& Sarma, A. K. (2007). A standardized test battery for the study of synesthesia. Journal of Neuroscience Methods, 159(1), 139-145.

First, M. B., \& Gibbon, M. (2004). The structured clinical interview for DSM-IV axis I disorders (SCID-I) and the structured clinical interview for DSM-IV axis II disorders (SCID-II). In Comprehensive Handbook of Psychological Assessment (Vol. 2).

Hannelius, U., Gherman, L., Mäkelä, V.-V., Lindstedt, A., Zucchelli, M., Lagerberg, C., Tybring, G., Kere, J., \& Lindgren, C. M. (2007). Large-scale zygosity testing using single nucleotide polymorphisms. Twin Research and Human Genetics, 10(04), 604625.

Hoekstra, R. A., Bartels, M., Cath, D. C., \& Boomsma, D. I. (2008). Factor structure, reliability and criterion validity of the Autism-Spectrum Quotient (AQ): A study in Dutch population and patient groups. Journal of Autism and Developmental Disorders, 38(8), 1555-1566.

Hoffman, S. N., Urosevich, T. G., Kirchner, H. Lester., Boscarino, J. J., Dugan, R. J., Withey, C. A., Adams, R. E., Figley, C. R., \& Boscarino, J. A. (2019). Grapheme-Color Synesthesia is Associated with PTSD Among Deployed Veterans: Confirmation of Previous Findings and Need for Additional Research. International Journal of Emergency Mental Health, 21(1). https://www.ncbi.nlm.nih.gov/pmc/articles/PMC6613655/

Houghton, D. C., Stein, D. J., \& Cortese, B. M. (2020). Review: Exteroceptive Sensory Abnormalities in Childhood and Adolescent Anxiety and Obsessive-Compulsive Disorder: A Critical Review. Journal of the American Academy of Child and Adolescent Psychiatry, 59(1), 78-87. https://doi.org/10.1016/j.jaac.2019.06.007

Hughes, J., Gruffydd, E., Simner, J., \& Ward, J. (2019). Synaesthetes show advantages in savant skill acquisition: Training calendar calculation in sequence-space synaesthesia. Cortex, 113, 67-82. https://doi.org/10.1016/j.cortex.2018.11.023

Hughes, J., Simner, J., Baron-Cohen, S., Treffert, D. A., \& Ward, J. (2017). Is synaesthesia more prevalent in autism spectrum conditions? Only where there is prodigious talent. 
Kamath, M. S., Dahm, C. R., Tucker, J. R., Huang-Pollock, C. L., Etter, N. M., \& Neely, K. A. (2020). Sensory profiles in adults with and without ADHD. Research in Developmental Disabilities, 104, 103696.

Kaufman, J., Birmaher, B., Brent, D., Rao, U. M. A., Flynn, C., Moreci, P., Williamson, D., \& Ryan, N. (1997). Schedule for affective disorders and schizophrenia for school-age children-present and lifetime version (K-SADS-PL): Initial reliability and validity data. Journal of the American Academy of Child \& Adolescent Psychiatry, 36(7), 980-988.

Kessler, J., Schaaf, A., \& Mielke, R. (1993). Der Fragmentierte Bildertest:(FBT). Hogrefe.

Kooij, J. J. S. (2010). Diagnostic interview for ADHD in adults 2.0 (DIVA 2.0). Adult ADHD. Diagnostic Assessment and Treatment. Pearson Assessment and Information BV, Amsterdam.

Lord, C., Rutter, M., DiLavore, P., Risi, S., Gotham, K., \& Bishop, S. (2012). Autism Diagnostic Observation Schedule-2nd Edition (ADOS-2). Los Angeles, CA: Western Psychological Corporation.

Lord, Catherine, Rutter, M., \& Le Couteur, A. (1994). Autism Diagnostic Interview-Revised: A revised version of a diagnostic interview for caregivers of individuals with possible pervasive developmental disorders. Journal of Autism and Developmental Disorders, 24(5), 659-685.

Luria, A. R., \& Solotaroff, L. T. (1987). The mind of a mnemonist: A little book about a vast memory. Harvard University Press.

Milne, E., Swettenham, J., Hansen, P., Campbell, R., Jeffries, H., \& Plaisted, K. (2002). High motion coherence thresholds in children with autism. Journal of Child Psychology and Psychiatry, 43(2), 255-263.

Mottron, L., Burack, J. A., larocci, G., Belleville, S., \& Enns, J. T. (2003). Locally oriented perception with intact global processing among adolescents with high-functioning autism: Evidence from multiple paradigms. Journal of Child Psychology and Psychiatry, 44(6), 904-913.

Mottron, L., Dawson, M., Soulieres, I., Hubert, B., \& Burack, J. (2006). Enhanced perceptual functioning in autism: An update, and eight principles of autistic perception. Journal of Autism and Developmental Disorders, 36(1), 27-43. 
Muth, A., Hönekopp, J., \& Falter, C. M. (2014). Visuo-spatial performance in autism: A metaanalysis. Journal of Autism and Developmental Disorders, 44(12), 3245-3263.

Neufeld, J., Roy, M., Zapf, A., Sinke, C., Emrich, H. M., Prox-Vagedes, V., Dillo, W., \& Zedler, M. (2013). Is synesthesia more common in patients with Asperger syndrome?

Pellicano, E., Gibson, L., Maybery, M., Durkin, K., \& Badcock, D. R. (2005). Abnormal global processing along the dorsal visual pathway in autism: A possible mechanism for weak visuospatial coherence? Neuropsychologia, 43(7), 1044-1053.

Robertson, A. E., \& Simmons, D. R. (2013). The relationship between sensory sensitivity and autistic traits in the general population. Journal of Autism and Developmental disorders, 43(4), 775-784.

Rothen, N., Seth, A. K., Witzel, C., \& Ward, J. (2013). Diagnosing synaesthesia with online colour pickers: Maximising sensitivity and specificity. Journal of Neuroscience Methods, 215(1), 156-160.

Ruzich, E., Allison, C., Smith, P., Watson, P., Auyeung, B., Ring, H., \& Baron-Cohen, S. (2015). Measuring autistic traits in the general population: a systematic review of the Autism-Spectrum Quotient (AQ) in a nonclinical population sample of 6,900 typical adult males and females. Molecular autism, 6(1), 2.

Shah, A., \& Frith, U. (1993). Why do autistic individuals show superior performance on the block design task? Journal of Child Psychology and Psychiatry, 34(8), 1351-1364.

Simner, J., Ward, J., Lanz, M., Jansari, A., Noonan, K., Glover, L., \& Oakley, D. A. (2005). Non-random associations of graphemes to colours in synaesthetic and nonsynaesthetic populations. Cognitive Neuropsychology, 22(8), 1069-1085.

Smilek, D., Dixon, M. J., \& Merikle, P. M. (2005). Synaesthesia: discordant male monozygotic twins. Neurocase, 11(5), 363-370.

Smilek, D., Moffatt, B. A., Pasternak, J., White, B. N., Dixon, M. D., \& Merikle, P. M. (2002). Synaesthesia: A case study of discordant monozygotic twins. Neurocase, 8(4), 338342.

Snodgrass, J. G., \& Corwin, J. (1988). Perceptual identification thresholds for 150 fragmented pictures from the Snodgrass and Vanderwart picture set. Perceptual and motor skills, 67(1), 3-36. 
Tilot, A. K., Vino, A., Kucera, K. S., Carmichael, D. A., van den Heuvel, L., den Hoed, J., Sidoroff-Dorso, A. V., Campbell, A., Porteous, D. J., St Pourcain, B., van Leeuwen, T. M., Ward, J., Rouw, R., Simner, J., \& Fisher, S. E. (2019). Investigating genetic links between grapheme-colour synaesthesia and neuropsychiatric traits. Philosophical Transactions of the Royal Society B: Biological Sciences, 374(1787), 20190026. https://doi.org/10.1098/rstb.2019.0026

Tilot, A., Kucera, K. S., Vino, A., Asher, J., Baron-Cohen, S., \& Fischer, S. (2018). Rare variants in axonogenesis genes connect three families with sound-color synesthesia. 223(1), 48-52. https://doi.org/10.1073/pnas.1715492115

Tomson, S. N., Avidan, N., Lee, K., Sarma, A. K., Tushe, R., Milewicz, D. M., Bray, M., Leal, S. M., \& Eagleman, D. M. (2011). The genetics of colored sequence synesthesia: Suggestive evidence of linkage to $16 q$ and genetic heterogeneity for the condition. Behavioural Brain Research, 223(1), 48-52.

Treffert, D. A. (2009). The savant syndrome: An extraordinary condition. A synopsis: past, present, future. Philosophical Transactions of the Royal Society B: Biological Sciences, 364(1522), 1351-1357. https://doi.org/10.1098/rstb.2008.0326

Van der Hallen, R., Evers, K., Brewaeys, K., Van den Noortgate, W., \& Wagemans, J. (2015). Global processing takes time: A meta-analysis on local-global visual processing in ASD. Psychological Bulletin, 141(3), 549.

Van Eylen, L., Boets, B., Steyaert, J., Wagemans, J., \& Noens, I. (2015). Local and global visual processing in autism spectrum disorders: Influence of task and sample characteristics and relation to symptom severity. Journal of Autism and Developmental Disorders, 1-23.

Van Leeuwen, T. M., van Petersen, E., Burghoorn, F., Dingemanse, M., \& van Lier, R. (2019). Autistic traits in synaesthesia: Atypical sensory sensitivity and enhanced perception of details. Philosophical Transactions of the Royal Society B: Biological Sciences, 374(1787), DOI: 20190024. https://doi.org/10.1098/rstb.2019.0024

Van Leeuwen, T.M., Neufeld, J., Hughes, J., \& Ward, J. (2020). Synaesthesia and Autism: Different Developmental Outcomes from Overlapping Mechanisms? Cognitive Neuropsychology. DOI: 10.1080/02643294.2020.1808455

Ward, J. (2013). Synesthesia. Annual Review of Psychology, 64, 49-75. 
Ward, J. (2019). Synaesthesia: A distinct entity that is an emergent feature of adaptive neurocognitive differences. Philosophical Transactions of the Royal Society $B$, 374(1787), 20180351.

Ward, J., Brown, P., Sherwood, J., \& Simner, J. (2017a). An autistic-like profile of attention and perception in synaesthesia. Cortex.

Ward, J., Field, A. P., \& Chin, T. (2019). A meta-analysis of memory ability in synaesthesia. Memory, 27(9), 1299-1312.

Ward, J., Hoadley, C., Hughes, J. E., Smith, P., Allison, C., Baron-Cohen, S., \& Simner, J. (2017b). Atypical sensory sensitivity as a shared feature between synaesthesia and autism. Scientific Reports, 7.

Ward, J., \& Simner, J. (2020). Chapter 13 - Synesthesia: The current state of the field. In K. Sathian \& V. S. Ramachandran (Eds.), Multisensory Perception (pp. 283-300). Academic Press. https://doi.org/10.1016/B978-0-12-812492-5.00013-9

Wechsler, D. (2003). WISC-IV Wechsler Intelligence Scale for Children: Technical and Interpretative: Manual. Pearson.

Wechsler, D. (2008). WAIS-IV: Wechsler Adult Intelligence Scale-4th Edition. San Antonio, TX: Pearson.

Wendler, E., \& Schubert, E. (2019). Synaesthesia, Creativity and Obsessive-Compulsive Disorder: Is There a Link? Creativity Research Journal, 31(3), 329-334. https://doi.org/10.1080/10400419.2019.1631637

Witkin, H. A. (1971). A manual for the embedded figures tests. Consulting Psychologists Press.

Witthoft, N., \& Winawer, J. (2006). Synesthetic colors determined by having colored refrigerator magnets in childhood. Cortex, 42(2), 175-183.

Witthoft, N., Winawer, J., \& Eagleman, D. M. (2015). Prevalence of learned grapheme-color pairings in a large online sample of synesthetes. PLoS One, 10(3), e0118996.

Zetterqvist, J., \& Sjölander, A. (2015). Doubly Robust Estimation with the R Package drgee. Epidemiologic Methods, 4(1), 69-86. 